\title{
Istoriato dell'elaborazione di linee guida sulle perizie psichiatriche assicurative, nelle regioni di lingua tedesca, ed in particolare in Svizzera
}

Le linee guida della Società Svizzera di Psichiatria Assicurativa, a proposito della valutazione peritale dei disturbi psichici

\author{
R. Marelli, presidente della Società Svizzera di Psichiatria Assicurativa
}

Deutsch erschienen in Nr. 20/2004

La version française a paru dans le $n^{\circ} 36 / 2004$

Corrispondenza:

Dr. med. Renato Marelli

Leonhardsstrasse 16

CH-4051 Basilea

Tel. 0612721821

Fax 0612612602

E-mail: renato.marelli@bluewin.ch
Il termine di «linee guida» («guidelines») designa il fatto di riunire e di valutare conoscenze medico-scientifiche ed esperienze empiriche secondo criteri coerenti, formulati in modo esplicito, da cui dedurne raccomandazioni per un'adeguata pratica clinica [1]. La Federazione dei medici svizzeri (FMH) ha dato importanza alle linee guida, ed ha definito delle regole per la loro elaborazione [2]. È principalmente in materia terapeutica che sono state formulate delle linee guida; le tematiche riguardanti il controllo di qualità delle perizie mediche, e particolarmente le perizie psichiatriche destinate alle assicurazioni, restano a lungo assenti dalla letteratura medica svizzera ed europea [3]. Linee guida dettagliate, sul tema della «perizia medica nella medicina psicosomatica e psicoterapeutica, nel campo del diritto delle assicurazioni sociali» sono disponibili [4], oggi, in Germania. Esse fanno tuttavia riferimento alla giurisprudenza tedesca, e non possono essere applicate tali e quali alle condizioni del nostro paese [5]. Da noi, Schneeberger [6] e Marelli [7] hanno già tempo fa evocato i problemi e le difficoltà sollevate dalla perizia psichiatrica in relazione con le assicurazioni sociali, in particolare quando si tratta di valutare dei disturbi somatoformi. È Fredenhagen [8] che per primo ha pubblicato uno studio completo che ingloba aspetti medici e giuridici della perizia medica. Nel capitolo dedicato alla psichiatria, Marelli trattava di questioni relative alla perizia psichiatrica richiesta da un'assicurazione, e formulava raccomandazioni che non pretendevano però d'assurgere alla dignità di linee direttive. Nel nostro paese mancano criteri di qualità riconosciuti. Per questo è stata fondata la Società Svizzera di Psichiatria Assicurativa (SSPA) nel 1996, molto prima che i problemi relativi all'invalidità assumessero al ruolo che occupano oggi nella politica, nei media e nella letteratura giuridica e medica [9].

\section{La Società Svizzera di Psichiatria Assicurativa}

I membri ordinari della SSPA sono specialisti in psichiatria e psicoterapia degli adulti o dei bambini. Essi dispongono di un'esperienza di parecchi anni nel campo delle perizie mediche richieste dalle assicurazioni. In qualità di società affiliata alla Società Svizzera di Psichiatria e Psicoterapia (SSPP), la SSPA fa propri i fini perseguiti dalla SSPP. Si occupa, in particolare, di formazione e di controllo della qualità nel suo campo specifico, ed organizza regolarmente incontri scientifici.

Una prima bozza dei criteri di qualità per una perizia psichiatrica è stata formulata già al momento della sua prima riunione annuale, nel 1997. L'incontro scientifico del 1998 è stato dedicato ai disturbi psicogeni che insorgono dopo un incidente. Si è così potuto discutere delle linee guida per la diagnosi dei Disturbi dell'adattamento, e dei Disturbi da stress post-traumatico. A partire dal 1999, la riflessione scientifica è proseguita grazie ad un quaderno di psichiatria assicurativa, pubblicato due volte l'anno, che ha proposto regolarmente una rubrica bibliografica sul tema della perizia medica. Nel 1999, la riunione annuale della società è stata dedicata specificamente al problema del controllo di qualità nelle perizie riguardanti i disturbi somatoformi. Klaus Foerster, di Tübingen, ha presentato una relazione sugli aspetti medici della questione, mentre Ulrich Meyer-Blaser, giudice del Tribunale federale delle assicurazioni, ha passato in rassegna le esigenze alle quali una perizia psichiatrica deve soddisfare per corrispondere alle attese del giurista chiamato a pronunciarsi sulle questioni correlate al concetto di esigibilità, nella valutazione di un'invalidità.

Il modo di effettuare le perizie psichiatriche richieste dalle assicurazioni è stato oggetto di 
un'inchiesta svolta tra i membri della SSPA. Quest'inchiesta ha mostrato una certa uniformità nel procedere all'esame dell'incarto, alla raccolta dell'anamnesi, all'osservazione clinica, ed alla valutazione, ma anche alcune divergenze, riguardanti il tempo dedicato ad una perizia, l'uso di test psicometrici, ed alcuni altri punti di minore importanza. Ne abbiamo concluso che bisognava definire dei criteri formali per la perizia psichiatrica nel campo assicurativo, se non vogliamo che ci venga rimproverato di lavorare in una maniera non scientifica.

\section{L'aumento del numero di beneficiari di rendite dell'assicurazione federale d'invalidità}

Nel 2000, non appena ha cominciato a evidenziarsi un ragguardevole aumento del numero di beneficiari di una rendita dell'assicurazione invalidità [10], ci è sembrato importante trattare approfonditamente i problemi della qualità nelle perizie AI. Beatrice Breitenmoser, vice-direttrice della Divisione Assicurazione invalidità dell'UFAS, ha parlato dell'importanza della perizia psichiatrica nell'assicurazione invalidità; Walter Böhni, psichiatra a Tann-Rüti, dei fattori determinanti per l'evoluzione, e Werner Durrer, direttore dell'ufficio AI di Lucerna, delle questioni relative al reinserimento professionale. Nel 2001, la giornata scientifica della società è stata di nuovo dedicata alla questione del controllo di qualità. Paul Hoff, allora direttore della Clinica universitaria di psichiatria e di psicoterapia di Aquisgrana, ha trattato delle esigenze qualitative in materia di diagnosi; Hans Georg Kopp, psichiatra alla Rehaklinik di Bellikon, della valutazione degli elementi clinici e diagnostici nella prospettiva della capacità di lavoro, e Thomas Weber, psichiatra a Basilea, delle trappole e degli errori in cui può cadere il perito psichiatrico nell'esercizio della sua attività.

\section{L'elaborazione di linee guida per le perizie mediche sui disturbi psichici, su incarico dalle assicurazioni}

Nel corso del 2002, il gruppo di lavoro sulle linee guida della SSPA, costituito da psichiatri in pratica privata o in ambito istituzionale, ha passato in rassegna ed analizzato le riflessioni e gli studi finora svolti dalla società. Ha completato gli aspetti della questione integrandovi i dati di diverse pubblicazioni dedicate alla perizia psichiatrica. Tra questi, menzioniamo l'articolo di Kind
[11] che descrive secondo quali criteri una perizia medica dovrebbe essere realizzata; quello di Gmür [12] che specifica nei dettagli le esigenze alle quali una perizia psichiatrica dovrebbe rispondere; quello di Meine [13], sull'aspetto relativo di un parere medico; quello di Ebner [14], dedicato alla perizia medico-sociale degli immigranti in Svizzera; quello di Grünig [15], che mette a fuoco i limiti giuridici della presa in considerazione delle perizie mediche nel diritto delle assicurazioni sociali; quello di Meine [16], che sottolinea il carattere completo della documentazione; ed i lavori più generali di Foerster [17] e di Rosatti [18] a proposito della perizia psichiatrica; di Schneider, Henningsen e Rüger [19] sulla perizia medico-sociale; e parimenti quello dedicato da Meyer-Blaser [20] e Mosimann [21] alla questione della collaborazione tra giudici e medici. Un'importanza particolare è stata accordata al catalogo di criteri elaborato dal Tribunale federale delle assicurazioni per l'apprezzamento di una perizia medica [22].

Tutti questi dati sono stati rivisti e precisati nel 2002, in occasione di una conferenza di consenso, alla quale erano stati invitati tutti i membri della SSPA. Il gruppo di lavoro sulle linee guida ne ha infine curato la redazione.

Nel 2003, il comitato della Società Svizzera di Psichiatria e Psicoterapia (SSPP) ha preso conoscenza delle linee guida, e le ha approvate, esprimendo la raccomandazione che tutti gli psichiatri attivi nel campo peritale dovrebbero esercitare anche un'attività clinica e terapeutica. È un'esigenza che deriva dal fatto che gli specialisti in psichiatria e psicoterapia devono soddisfare alle esigenze della SSPP in materia di formazione continua, che richiedono l'esercizio di un'attività clinica-terapeutica. Il 13 novembre 2003, le linee guida sono state approvate e quindi ufficializzate dall'Assemblea generale della SSPA.

\section{Reazioni da parte del pubblico interessato}

Non appena pubblicate, le linee guida hanno suscitato subito un grande interesse. Gli psichiatri hanno accolto favorevolmente le raccomandazioni formali per la strutturazione delle perizie nel campo delle assicurazioni sociali, mentre i giuristi hanno apprezzato innanzi tutto le riflessioni che precisano il ruolo ed il campo di validità della perizia psichiatrica nel contesto del diritto assicurativo, e le osservazioni che sottolineano le differenze tra i modi di pensare normativo del giurista, e quelle che derivano dall'approccio medico, orientate verso l'osservazione empirica. Meyer-Blaser [5] è del parere che parec- 
chie esigenze formulate dalle linee direttive siano di natura tale da contribuire ad un miglioramento della qualità delle perizie, ossia, da parte dei periti, ad una migliore considerazione delle nozioni giuridiche fondamentali.

Con la pubblicazione nel Bollettino dei Medici Svizzeri delle prime linee guida elaborate in Svizzera sul tema della perizia psichiatrica nel campo delle assicurazioni, la SSPA intende metterle a disposizione dei colleghi attivi nel campo, e delle persone interessate a questioni riguardanti il diritto delle assicurazioni sociali. Si tratta, in particolare, dei giuristi dell'amministrazione e dei tribunali, a livello cantonale, ed a livello del Tribunale Federale delle Assicurazioni.

\section{Riferenze}

1 Obrist R. Guidelines. Was sie sollten und was sie tun. Schweiz Ärztezeitung 2001;82(24):1278-81.

2 Eicher E. Guidelines für Guidelines. Schweiz Ärztezeitung 1999;80:581-3.

3 Winckler P, Foerster K. Qualitätskriterien in der psychiatrischen Begutachtungspraxis. Versicherungsmedizin 1994;46:49-52.

4 Arbeitsgemeinschaft wissenschaftlich-medizinischer Fachgesellschaften (AWMF), raggiungibile in Internet nel sito http://www.uni-duesseldorf.de/ WWW/AWMF/.

5 Meyer-Blaser U. Der Rechtsbegriff der Arbeitsunfähigkeit und seine Bedeutung in der Sozialversicherung, namentlich für den Einkommensvergleich in der Invaliditätsbemessung. In: Schaffhauser R, Schlauri F. Schmerz und Arbeitsunfähigkeit. St. Gallen: Schriftenreihe des Instituts für Rechtswissenschaft und Rechtspraxis der IRP-HSG. 2003. p. 27-119.

6 Schneeberger E. Die psychiatrische Beurteilung von Rentenanwärtern. ZAK 1986;203-9.

7 Marelli R. Weichteilschmerzen und psychiatrische Begutachtung. ZAK 1991;155-65.

8 Fredenhagen H. Das ärztliche Gutachten. 3. vollständig überarbeitete Auflage. Bern: Huber; 1994.
9 Saurer A, Davoine GA, Godinat G, Petite D. Crise socio-économique et évaluation de l'incapacité de travail de longue durée. SZS 2000;44:1-13.

10 Breitenmoser B, Foffa D, Guggisberg K, Rouiller C, Donini F, Nydegger Lory B. Eingliederung vor Rente - realisierbares Ziel oder bloss wohltönender Slogan? Soziale Sicherheit 1999:288-92.

11 Kind H. So entsteht ein medizinisches Gutachten. In: Schaffhauser R, Schlauri H. Rechtsfragen der medizinischen Begutachtung in der Sozialversicherung. St. Gallen. Bd 42 der Veröffentlichungen des Schweiz. Instituts für Verwaltungskurse 1997. p. $49-67$.

12 Gmür M. Die Anforderungen an psychiatrische Gutachten. Plädoyer 1999;(4):28-44.

13 Meine J. Die Bedingtheit der ärztlichen Aussage. SZS 1999;44(2):89-97.

14 Ebner G. Sozialmedizinische Begutachtung von Migrantinnen und Migranten in der Schweiz. Psychiatrie 2002;2:20-5.

15 Grünig C. Medizinische Gutachten in Rechtsfällen: Mitteilungen der Lebensversicherer an die Ärzteschaft. Schweiz. Versicherungsverband 2002; 4-13.

16 Meine J. L'expertise médicale en Suisse: satisfaitelle aux exigences de qualité actuelle? Rev Suisse d'Assurances 1999;67:7-45.

17 Foerster K. Psychiatrische Begutachtung im Sozialrecht. In: Foerster K, Venzlaff U. Psychiatrische Begutachtung. 3., neu bearbeitete Auflage. München-Jena: Urban \& Fischer; 2000. p. 505-20.

18 Rosatti P. L'expertise médicale. Genève. Médecine \& Hygiène; 2002.

19 Schneider W, Henningsen P, Rüger U. Sozialmedizinische Begutachtung in Psychosomatik und Psychotherapie, 1. Auflage. Bern: Huber; 2003.

20 Meyer-Blaser U. Sozialversicherungsrecht und Medizin. In: Fredenhagen H. Das ärztliche Gutachten. 4. Auflage. Bern: Huber; 2003.

21 Mosimann HJ. Somatoforme Störungen: Gerichte und (psychiatrische) Gutachten. SZS 1999; 43:1-21 und 105-128.

22 BGE 125 V 351ff. Erw. 3 mit zahlreichen Hinweisen, RKUV 2000 KV 124; 214. 